\title{
Signaling pathways underlying striatal synaptic plasticity and reward learning Kim T Blackwell
}

Address: George Mason University, Molecular Neuroscience Department, The Krasnow Institute for Advanced Study, Fairfax, VA, USA

Email: Kim T Blackwell - kblackw1@gmu.edu

from Seventeenth Annual Computational Neuroscience Meeting: CNS*2008

Portland, OR, USA. 19-24 July 2008

Published: II July 2008

BMC Neuroscience 2008, 9(Suppl I):L3 doi: 10.1 186/I47I-2202-9-SI-L3

This abstract is available from: http://www.biomedcentral.com/I47I-2202/9/SI/L3

(c) 2008 Blackwell; licensee BioMed Central Ltd.

Operant conditioning is a form of associative learning in which rewarding an animal's response increases the likelihood of eliciting the response. The ability to use appropriately timed rewards to shape complex behaviour inspires scientists in psychology, neurophysiology, and modeling. Temporal difference models and experimental results agree that reward elicits dopamine release in the striatum and that striatal spiny projection neurons learn the association between the motor response and reward. Nonetheless, critical aspects of operant conditioning behavior have not been replicated. In particular, if synaptic plasticity underlies learning, then the temporal interval between dopamine and cortical inputs should be critical in producing plasticity of cortico-striatal synapses. To investigate the mechanisms whereby glutamate and dopamine interact to produce plasticity, we develop a computer model of the signaling pathways activated by dopamine and glutamate in the spiny projection neuron of the striatum. In the model, dopamine activates adenylyl cyclase, which produces the diffusible molecule, CAMP, which binds to PKA. Glutamate produces an elevation in intracellular calcium, which binds to calmodulin and activates CaMKII. These pathways interact through DARPP32. Model simulations show that simultaneous dopamine and glutamate produce a synergistic increase in PKA activation and DARPP32 phosphorylation consistent with the requirement for both dopamine and glutamate for learning behavior. We are presently developing a multicompartment model in which the reactions and diffusion implicit in these signaling pathways are simulated stochastically in the spines along a dendrite. Using this model we plan to investigate the spatio-temporal patterns of synaptic input that produce an elevation in critical enzymes, and to test whether plasticity is sensitive to temporal interval. 